\title{
Recommandations pratiques concernant la sortie des patients après traitement du cancer différencié de la thyroïde à l ${ }^{, 131}$ I
}

\author{
T. CARLIER ${ }^{1}$, A. LISBONA ${ }^{2}$, F. KRAEBER-BODERE ${ }^{1,3}$, C. ANSQUER $^{1}$, \\ O. COUTURIER ${ }^{1,3}$
}

(Manuscrit reçu le 18 février 2004, accepté le 22 mai 2004)

RÉSUMÉ Peu d'études sur le thème des recommandations de radioprotection que doivent suivre les patients hors du secteur d'hospitalisation après traitement du cancer différencié de la thyroïde par de l'iode-131, sont basées sur une détermination expérimentale de la période effective. Le but de ce travail a été de calculer des valeurs de durées pendant lesquelles le patient doit respecter des recommandations à l'aide d'une mesure du débit de dose à $30 \mathrm{~cm}$ le dernier jour avant qu'il quitte le secteur d'hospitalisation. Les mesures du débit de dose ont été réalisées tous les jours au niveau de l'abdomen et du cou pendant la durée d'hospitalisation. Ces points sont ajustés par un modèle mono-exponentiel ou bi-exponentiel pour déterminer la période effective. Nous modélisons 7 situations d'exposition des personnes proches. Nous montrons que dans les conditions réalistes de diffusion le rapport des débits de dose en fonction de la distance suit une loi en l'inverse de la distance de mesure. La période effective est de $16 \pm 6$ h quelle que soit la localisation de la mesure. Cela nous conduit à un calcul de respect de recommandations n'excédant pas 2 jours après 3 jours d'hospitalisation. Un abaque simple est donné pour permettre le calcul des durées de recommandations en fonction du débit de dose mesuré à la sortie. Ce protocole de mesure est mise en oeuvre dans notre institution et donne entière satisfaction tant du point de vue de l'information au patient que du respect de l'irradiation minimale des proches suivant le principe ALARA.

ABSTRACT Pratical recommandations for outpatients after differentiated thyroid carcinoma treatment with iodine-131.

Few studies on radiation protection recommandation for patients leaving hospitalisation area after thyroid carcinoma treatment with iodine-131 are based on experimental determination of effective half life. The aim of this work was to calculate times of adhesion to restrictions for patients using one dose rate measurement at $30 \mathrm{~cm}$ the last day, just before leaving hospital. Dose rate measurements were achieved every day at two locatizations (abdomen mid trunk and neck) during hospitalisation. Datas were fitted by a monoexponential or a biexponential model in order to derive the effective half life. We condidered 7 exposure states between patient and neighbooring. We showed, according to our realistic scatter state, a dose rate variation inversely proportional to the distance from patient. Effective half life is equal to $16 \pm 6 \mathrm{~h}$ whatever the measurement localization. Thus the times of adhesion to restrictions is never greater than 2 days

\footnotetext{
Service de Médecine Nucléaire, CHU Hôtel Dieu, 1 place Alexis Ricordeau, 44035 Nantes, France.

Service de Physique Médicale, CRLCC René Gauducheau, Boulevard J. Monod, 44805 Saint-Herblain, France.

INSERM U601, 30 boulevard Jean Monnet, 44093 Nantes, France.
} 
after 3 days of hospitalisation. A simple table is given to allow the computation of restrictions times according to the dose rate measurement before the patient is discharged. This protocol is applied in our institution and gives whole satisfaction as far as the ALARA principle and the patient information are concerned.

\section{Introduction}

Les patients traités à l'iode-131 (on ne considère ici que les traitements des cancers différenciés de la thyroïde) quittent le secteur d'hospitalisation pour, dans la plupart des cas, un retour à leur domicile. L'entourage direct du patient peut être ainsi amené à être exposé à des niveaux d'exposition significatifs. Les directives Euratom 96/29 (CEC, 1996) et 97/43 (CEC, 1997), récemment transposées dans la législation française (décret, 2002 ; décret, 2003 ; arrêté, 2004), offrent un cadre légal à la protection des personnes en contact avec le patient; en accord avec les principes généraux de radioprotection édictés, en particulier, par les publications 60 (ICRP, 1991) et 73 (ICRP, 1996) de la CIPR. Le but de cette étude préliminaire est de fournir un abaque simple donnant des périodes de respect de restriction de contact entre le patient et son entourage. Le paramètre permettant de donner les périodes de restriction est une mesure du débit de dose à $30 \mathrm{~cm}$ réalisée à la sortie du secteur d'hospitalisation.

Les valeurs des différentes durées de restriction en fonction de la mesure du débit de dose à $30 \mathrm{~cm}$ sont calculées à partir d'une évaluation expérimentale de la période effective et de modélisations des durées de contact.

\section{Matériels et méthodes}

Vingt-neuf patients ont été inclus dans cette étude. Nous distinguons les patients bénéficiant pour la première fois du traitement (patient $\mathrm{P}$ ) de ceux pour qui c'est le deuxième ou plus (patient $\mathrm{M}$ ). Deux patients ont une ou plusieurs fixations pulmonaires, une patiente présente une fixation surrénalienne et quatre patients ont une fixation cervicale supérieure à $5 \%$. De même, nous comptabilisons 10 patients M et 19 patients $P$.

\subsection{Détermination de la période effective $T_{\mathrm{eff}}$}

La période effective $T_{\text {eff }}$ est évaluée à l'aide de mesures réalisées à plusieurs distances patient-détecteur afin de minimiser les erreurs de mesures.

Nous réalisons une mesure du débit de dose à trois distances $(10 \mathrm{~cm}, 30 \mathrm{~cm}$ et $100 \mathrm{~cm}$ ) tous les jours (l'heure de mesure étant notée précisément) pendant la période d'hospitalisation (5 jours dans notre institution), dans les mêmes 
conditions géométriques (patient debout). Nous utilisons un radiamètre ThermoEberline ESM FH 40 G-L10 étalonné. Les mesures sont réalisées au niveau de l'abdomen (vessie vide) pour rendre compte de l'activité non spécifique de l'iode. Un deuxième site de mesure est choisi au niveau du cou (sauf pour la mesure à $100 \mathrm{~cm}$ ) exprimant la fixation spécifique d'éventuels reliquats thyroïdiens. Il n'a pas été fait de différence entre une faible et importante fixation cervicale. Le but est en effet d'estimer la cinétique d'élimination indépendamment de la quantité fixée.

L'avantage de cette méthode est de prendre en compte les phénomènes d'atténuation et de diffusion dus au patient.

La détermination de la période effective se fait par ajustement des points expérimentaux à l'aide d'un modèle mathématique en utilisant l'algorithme de Levenberg-Marquardt. Nous choisissons d'évaluer un modèle mono-exponentiel donnant une valeur $T_{\text {eff }}^{\text {mono }}$ et un modèle bi-exponentiel donnant deux valeurs $T_{\text {eff }}^{\mathrm{bi}^{1}}$ et $T_{\mathrm{eff}}^{\mathrm{bi}^{2}}$. Ces deux modèles sont évalués afin de mettre en évidence un éventuel comportement à deux cinétiques dans l'élimination de l'iode-131. Le choix de l'un ou l'autre des modèles se fait suivant deux critères :

1. si $T_{\mathrm{eff}}^{\mathrm{bi}^{1}}=T_{\mathrm{eff}}^{\mathrm{bi}}=T_{\text {eff }}^{\text {mono }}$ alors nous choisissons le modèle mono-exponentiel ;

2. dans les autres cas, nous choisissons le modèle qui ajuste au mieux les données expérimentales par comparaison des coefficients non-diagonaux de la matrice de variance-covariance.

\subsection{Modélisation et calcul des périodes de respect des recommandations}

Plusieurs situations pratiques d'exposition (décrites dans le Tab. I) sont envisagées afin de couvrir au mieux la vie courante. Elles sont choisies en partie à partir du travail de Barrington (Barrington et al., 2002). Ces situations sont assorties d'une limite maximale de dose efficace $D_{\mathrm{T}}^{\max }$ en accord avec les recommandations d'un rapport de la Commission de la communauté européenne (1999) afin de tenir compte de l'âge et de toutes les autres formes d'irradiation additionnelle. Nous supposons par ailleurs, que la contribution à la dose efficace est négligeable pour des distances supérieures à 1 mètre.

\subsubsection{Calcul par le modèle mono-exponentiel}

Comme mentionné précédemment, le calcul repose sur la mesure du débit de dose à $30 \mathrm{~cm} \dot{D}_{\text {ini }}$ juste avant la sortie du patient du secteur d'hospitalisation.

On suppose une durée d'irradiation fixe égale à $\Delta t=t_{\text {fin }}-t_{\text {ini }}$ avec $t_{\text {ini }}=0$ (toutes les durées sont exprimées en jour). 
TABLEAU I

Description des situations pratiques et limites de doses associées. Description of practical exposition situations and associated dose limits.

\begin{tabular}{|c|c|c|c|}
\hline Cas & Description de la situation & $\begin{array}{c}\text { Temps de modélisation } \\
\text { (par jour) }\end{array}$ & $D_{\mathrm{T}}^{\max }(\mathbf{m S v})$ \\
\hline A & Contact avec le (la) conjoint(e) & $8 \mathrm{~h}$ à $0,3 \mathrm{~m}+6 \mathrm{~h}$ à $1 \mathrm{~m}$ & $\begin{array}{c}3 \text { (si âge }<60 \text { ans }) \\
15 \text { (si âge }>60 \text { ans) }\end{array}$ \\
\hline B & Transport en commun & $30 \min$ à $0,1 \mathrm{~m}$ & 0,3 \\
\hline $\mathrm{C}$ & $\begin{array}{c}\text { Contact avec un enfant }(<2 \text { ans) au retour } \\
\text { à la maison }\end{array}$ & $9 \mathrm{~h}$ à $0,1 \mathrm{~m}$ & 1 \\
\hline $\mathrm{D}$ & $\begin{array}{c}\text { Contact avec un enfant ( } \geq 2 \text { ans et }<5 \text { ans) } \\
\text { au retour à la maison }\end{array}$ & $4 \mathrm{~h}$ à $0,1 \mathrm{~m}+8 \mathrm{~h}$ à $1 \mathrm{~m}$ & 1 \\
\hline $\mathrm{E}$ & $\begin{array}{c}\text { Contact avec un enfant ( } \geq 5 \text { ans et }<11 \text { ans) } \\
\text { au retour à la maison }\end{array}$ & $2 \mathrm{~h}$ à $0,1 \mathrm{~m}+4 \mathrm{~h}$ à $1 \mathrm{~m}$ & 1 \\
\hline $\mathrm{F}$ & Contact avec une femme enceinte & $6 \mathrm{~h}$ à $1 \mathrm{~m}$ & 1 \\
\hline $\mathrm{G}$ & Contact avec des collègues de travail & $8 \mathrm{~h}$ à $1 \mathrm{~m}$ & 0,3 \\
\hline
\end{tabular}

La dose efficace corps entier sur $n$ jours est donnée par la relation suivante :

$$
\begin{gathered}
D_{\mathrm{T}}^{\mathrm{max}}=\dot{D}_{\mathrm{ini}} \int_{t_{\text {ini }}}^{t_{\text {fin }}} \exp \left(-\frac{\ln 2}{T_{\text {eff }}^{\text {mono }}} t\right) d t \sum_{k=0}^{n} \exp \left(-\frac{k \ln 2}{T_{\text {eff }}^{\text {mono }}}\right) \\
\Leftrightarrow D_{\mathrm{T}}^{\max }=\dot{D}_{\text {ini }} \frac{T_{\text {eff }}^{\text {mono }}}{\ln 2}\left(1-\exp \left(-\frac{\ln 2}{T_{\text {eff }}^{\text {mono }}} \Delta t\right)\right) \frac{1-\exp \left(-\frac{(n+1) \ln 2}{T_{\text {eff }}^{\text {mono }}}\right)}{1-\exp \left(-\frac{\ln 2}{T_{\text {eff }}^{\text {mono }}}\right)} .
\end{gathered}
$$

On considérera toujours une irradiation des proches sur un temps infini. La relation précédente devient donc :

$$
D_{\mathrm{T}}^{\mathrm{max}}=\dot{D}_{\mathrm{ini}} \underbrace{\frac{T_{\text {eff }}^{\text {mono }}}{\ln 2}\left(1-\exp \left(-\frac{\ln 2}{T_{\text {eff }}^{\text {mono }}} \Delta t\right)\right) \frac{1}{1-\exp \left(-\frac{\ln 2}{T_{\text {eff }}^{\text {mono }}}\right)}} .
$$

$f(d)$

La fonction $f$ est dépendante de la distance de mesure $d$ en raison des incertitudes liées à la mesure. 
Le calcul du débit de dose minimal $\dot{D}_{\text {ini }}^{\text {min }}$ à $30 \mathrm{~cm}$ à la sortie du patient qui conduira à la dose efficace limite $D_{\mathrm{T}}^{\max }$ est donc donné par la relation suivante :

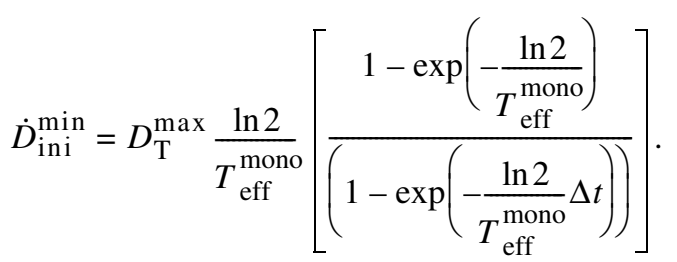

Le temps $t_{\text {lim }}$ pendant lequel le patient devra attendre de façon à ce que le débit de dose à $30 \mathrm{~cm}$ atteigne la valeur minimale qui conduira à la dose efficace limite pour ses proches est donc donné par la relation :

$$
t_{\lim }=\frac{T_{\mathrm{eff}}^{\mathrm{mono}}}{\ln 2} \ln \left(\frac{\dot{D}_{\mathrm{ini}}^{\mathrm{min}}}{\dot{D}_{\mathrm{ini}}}\right) .
$$

Ces relations sont valables pour des distances identiques à la distance de mesure initiale $(30 \mathrm{~cm})$.

\subsubsection{Calcul par le modèle bi-exponentiel}

On se ramène au cas mono-exponentiel car l'une des deux périodes effectives est, en pratique, très supérieure à l'autre. Au bout de 3 jours d'hospitalisation la contribution de l'un des facteurs exponentiels de la fonction est nettement inférieure à l'autre (d'un facteur 80 à 1000 : résultat non présenté dans cette étude). Ainsi, à la sortie du patient, nous ne tiendrons compte que d'une seule exponentielle.

Notons $T_{\mathrm{eff}}^{\mathrm{bi}}{ }^{\mathrm{max}}$ la plus grande des périodes effectives. Le temps $t_{\text {lim }}$ est donné de façon similaire par la relation :

$$
t_{\lim }=\frac{T_{\mathrm{eff}}^{\mathrm{bi}^{\max }}}{\ln 2} \ln \left(\frac{\dot{D}_{\mathrm{ini}}^{\min }}{\dot{D}_{\mathrm{ini}}}\right) .
$$

\subsubsection{Simplification du raisonnement dans le cas d'une modélisation à deux distances}

Le raisonnement précédent n'est valable que lorsque l'on est capable de se ramener de la distance de mesure à la sortie du patient $(30 \mathrm{~cm})$ à une distance $x$ (ici, $x=100 \mathrm{~cm}$ ou $x=10 \mathrm{~cm})$.

Nous avons le choix d'utiliser :

1. l'approximation standard de l'inverse carré des distances ; 
2. les données publiées par Siegel (Siegel et al., 2002a) (en assimilant le patient à une ligne source) ;

3. le rapport des mesures entre les valeurs du débit de dose à $30 \mathrm{~cm}$ et à la distance $x$.

Ainsi, dans les cas de modélisation à deux distances $d_{1}$ et $d_{2}$ (cas $\mathrm{A}, \mathrm{D}$ et $\mathrm{E}$ ); la relation (3) s'écrit :

$$
D_{\mathrm{T}}^{\max }=\dot{D}_{\text {ini }}^{d_{1}} f\left(d_{1}\right)+\dot{D}_{\text {ini }}^{d_{2}} f\left(d_{2}\right) .
$$

Or, le débit de dose à la distance $d_{2}$ est lié au débit de dose à la distance $d_{1}$ par la relation de proportionnalité suivante :

$$
\dot{D}_{\text {ini }}^{d_{2}}=k \dot{D}_{\text {ini }}^{d_{1}}
$$

Avec, $k$ facteur dépendant de la méthode choisie parmi les trois critères pour se ramener de la distance $d_{2}$ à la distance $d_{1}$.

L'équation (7) s'écrit donc :

$$
D_{\mathrm{T}}^{\max }=\dot{D}_{\mathrm{ini}}^{d_{1}}\left(f\left(d_{1}\right)+k f\left(d_{2}\right)\right) .
$$

L'équation (4) se ré-écrit :

$$
\dot{D}_{\mathrm{ini}}^{\min }=D_{\mathrm{T}}^{\max } \frac{1}{f\left(d_{1}\right)+k f\left(d_{2}\right)} .
$$

Nous écartons d'emblée la méthode de l'inverse carré des distances en raison de la violation de l'hypothèse de source ponctuelle (répartition étendue de l'activité dans le patient).

\section{Résultats}

\section{1. Évaluation du facteur $k$}

Les valeurs moyennes mesurées du facteur $k$ sont regroupées dans le tableau II.

Les calculs théoriques de Siegel (Siegel et al., 2002a) donnent des valeurs de $k$ allant de 10,7 (longueur de la ligne source : $20 \mathrm{~cm}$ ) à 5,8 (longueur de la ligne source : $174 \mathrm{~cm}$ ) pour se ramener d'une distance de mesure de $100 \mathrm{~cm}$ à $30 \mathrm{~cm}$; et de 7,3 à 3,5 pour se ramener d'une distance de mesure de $30 \mathrm{~cm}$ à $10 \mathrm{~cm}$.

Nos résultats nous conduisent plutôt à proposer de façon empirique une loi approximative en $d^{-1}$ pour le facteur $k$, où $d$ est la distance source-détecteur. 
TABLEAU II

Valeurs expérimentales moyennes du coefficient $k$. Mean experimental values of $k$ coefficient.

\begin{tabular}{lcc}
\hline Rapport des distances & $\boldsymbol{k}$ & Écart type \\
\hline De $100 \mathrm{~cm}$ à $30 \mathrm{~cm}$ (abdomen) & 3,4 & 0,7 \\
De $100 \mathrm{~cm}$ à $30 \mathrm{~cm}$ (cou) & 4,2 & 0,9 \\
De $30 \mathrm{~cm}$ à $10 \mathrm{~cm}$ (abdomen) & 2,3 & 0,4 \\
De $30 \mathrm{~cm}$ à $10 \mathrm{~cm}$ (cou) & 2,4 & 0,6 \\
\hline
\end{tabular}

TABLEAU III

Périodes effectives moyennes pour les mesures à l'abdomen. Mean effective half-life for abdomen measurements.

\begin{tabular}{ccc}
\hline \multirow{2}{*}{ Distance de mesure (cm) } & \multicolumn{2}{c}{ Période effective (h) } \\
\cline { 2 - 3 } & Patient M & Patient P \\
\hline 100 & $16 \pm 3$ & $16 \pm 6$ \\
30 & $15 \pm 3$ & $17 \pm 7$ \\
10 & $18 \pm 5$ & $17 \pm 6$ \\
\hline
\end{tabular}

TABLEAU IV

Périodes effectives moyennes pour les mesures cervicales. Mean effective half-life for cervical measurements.

\begin{tabular}{ccc}
\hline \multirow{2}{*}{ Distance de mesure $(\mathbf{c m})$} & \multicolumn{2}{c}{ Période effective (h) } \\
\cline { 2 - 3 } & Patient M & Patient P \\
\hline 30 & $15 \pm 3$ & $16 \pm 7$ \\
10 & $16 \pm 3$ & $17 \pm 7$ \\
\hline
\end{tabular}

Les valeurs que nous obtenons (en particulier pour passer de $100 \mathrm{~cm}$ à $30 \mathrm{~cm}$ ) sont cohérentes avec celles déjà publiées pour des cancers de la thyroïde (Culver et al., 1992) ainsi que pour des traitements de radioimmunothérapie à l'iode-131 (Siegel et al., 2002b ; Gates et al., 1998) ; où, en première approximation, la répartition du radioélément dans le corps est sensiblement identique et majoritairement localisée dans l'abdomen.

\subsection{Période effective}

Les périodes effectives en fonction de la distance et de la catégorie du patient (M ou P) sont regroupées dans le tableau III pour les mesures à l'abdomen et le tableau IV pour les mesures à la thyroïde. 
TABLEAU V

Période recommandée $t_{\text {lim }}$ en jours.

Recommended duration $t_{\lim }$ in days.

\begin{tabular}{ccccccc}
\hline \multirow{2}{*}{ Cas } & \multicolumn{5}{c}{ Débit de dose $\dot{D}_{\text {ini }}$ mesuré à la sortie du patient $\left(\mu \mathrm{Sv} . \mathrm{h}^{-1}\right)$} \\
\cline { 2 - 7 } & $200 \leq \dot{D}_{\text {ini }}<180$ & $180 \leq \dot{D}_{\text {ini }}<170$ & $170 \leq \dot{D}_{\text {ini }}<160$ & $160 \leq \dot{D}_{\text {ini }}<90$ & $90 \leq \dot{D}_{\text {ini }}<50$ & $\dot{D}_{\text {ini }} \leq 50$ \\
\hline $\begin{array}{c}\text { A (âge } \\
<60 \text { ans) }\end{array}$ & 0 & 0 & 0 & 0 & 0 & 0 \\
$\begin{array}{c}\text { A (âge } \\
>60 \text { ans) }\end{array}$ & 0 & 0 & 0 & 0 & 0 & 0 \\
B & 0 & 0 & 0 & 0 & 0 & 0 \\
C & 2 & 2 & 1 & 1 & 1 & 0 \\
D & 1 & 1 & 1 & 1 & 0 & 0 \\
E & 1 & 0 & 0 & 0 & 0 & 0 \\
F & 0 & 0 & 0 & 0 & 0 & 0 \\
G & 1 & 1 & 0 & 0 & 0 & 0 \\
\hline
\end{tabular}

Nous n'avons pas mesuré le débit de dose à $1 \mathrm{~m}$ au niveau de la thyroïde en raison de la réponse angulaire du radiamètre proche de l'unité pour une plage s'étendant de $-45^{\circ}$ à $+45^{\circ}$.

\subsection{Calcul des périodes de recommandations}

L'utilisation conjointe des paramètres donnés dans le tableau I ainsi que des relations (5) et (6) permet de calculer les durées de restriction de contact avec l'entourage suivant les situations envisagées. Un test statistique de Mann et Whitney est effectué entre les périodes effectives calculées pour des mesures à l'abdomen (Tab. III) et à la région cervicale (Tab. IV) ainsi qu'entre les périodes effectives trouvées pour les patients $\mathrm{P}$ et M. Quelle que soit la distance de mesure, les périodes effectives calculées entre les patients $\mathrm{P}$ et $\mathrm{M}$ ainsi que celles trouvées entre l'abdomen et la région cervicale ne sont pas différentes $(p>0,05)$. Nous choisissons donc une période effective de $16 \mathrm{~h}$. Les résultats sont donnés dans le tableau $\mathrm{V}$ pour des mesures de débit de dose à $30 \mathrm{~cm}$ caractéristique d'une hospitalisation de 3 jours. Les périodes de restriction sont données par valeurs entières supérieures.

Nous donnons en complément (de façon à pouvoir appliquer les calculs au

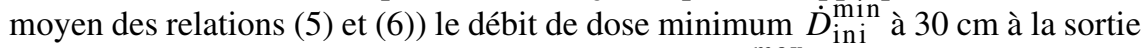
du patient qui conduira à la dose efficace limite $D_{\mathrm{T}}^{\max }$ en fonction de chaque situation envisagée (Tab. VI). 
TABLEAU VI

Débit de dose minimum mesuré à $30 \mathrm{~cm}$ conduisant à la dose efficace maximale $D_{\mathrm{T}}^{\max }$ en fonction des situations pratiques décrites dans le tableau $I$.

Minimum dose rate measured at $30 \mathrm{~cm}$ leading the maximum effective dose $D_{\mathrm{T}}^{\text {max }}$ according to practical exposition situation described in table $\mathrm{I}$.

\begin{tabular}{|c|c|}
\hline Cas & $\dot{D}_{\mathrm{ini}}^{\min }\left(\mu \mathrm{Sv} \cdot \mathrm{h}^{-1}\right)$ \\
\hline $\mathrm{A}$ (âge $<60$ ans) & 233 \\
\hline $\mathrm{A}$ (âge $>60$ ans) & 1164 \\
\hline B & 546 \\
\hline $\mathrm{C}$ & 36 \\
\hline D & 61 \\
\hline E & 116 \\
\hline $\mathrm{F}$ & 455 \\
\hline G & 107 \\
\hline
\end{tabular}

\section{Discussion}

La valeur de la mesure du débit de dose à $30 \mathrm{~cm}$ est en pratique la plus significative des mesures à l'abdomen et à la région cervicale. Certains patients présentent en effet des captations, au niveau du cou, plus élevées que d'autres conduisant à un débit de dose à la région cervicale supérieur à celui mesuré à l'abdomen.

Les durées de restriction mentionnées dans le tableau $\mathrm{V}$ appellent plusieurs remarques.

1. Les valeurs que nous avons trouvées sont globalement inférieures aux études menées précédemment sur ce sujet (Barrington et al., 2002 ; BIR, 1999; Monsieurs et al., 1998), sauf pour l'étude de Culver (Culver et al., 1992). L'étude de Monsieurs (Monsieurs et al., 1998) préconise des durées, pour des situations d'exposition sensiblement supérieures à celles que nous avons envisagées, de l'ordre de 7 jours (après 2 à 3 jours d'hospitalisation) quelque soit la quantité de radioélément présente dans l'organisme à la sortie du patient. La philosophie de cette étude repose sur une lecture de dosimètre thermoluminescent donné à l'entourage proche du patient. Par ailleurs, Monsieurs montre que pour une durée d'hospitalisation de 4 jours et aucune durée de restriction de contact donnée ; la dose efficace reçue par l'entourage n'a dépassé que très rarement ( 2 cas sur 19$)$ et très légèrement la valeur limite de $1 \mathrm{mSv}$. Ces résultats semblent aller dans le sens de nos conclusions.

Il est cependant plus surprenant que les valeurs de Barrington (Barrington et al., 2002) soient tout à fait différentes des nôtres (période de restriction variant de 10 à 24 jours) pour les patients bénéficiant d'une première 
administration thérapeutique, ce malgré la même méthodologie de mesure. Ceci est dû à l'évaluation de la période effective plus importante dans cette étude (3,6 jours en moyenne) que la nôtre (16 h). Guedj (Guedj et al., 2003) rapporte également une période effective plus importante $(2,1$ jours) mais à partir de seulement deux points de mesures sans distinguer les patients $\mathrm{P}$ et $\mathrm{M}$. De même, le rapport 53 de la CIPR (ICRP, 1988) mentionne une période effective pour l'activité non spécifique de 0,3 jours et de 7,3 jours pour l'activité spécifique. Nous pouvons en partie expliquer ces différences par le fait que nous n'avons pas poursuivi des mesures de débit de dose au-delà de la durée d'hospitalisation comme l'ont fait d'autres auteurs (Barrington et al., 2002 ; Guedj et al., 2003). Il faut cependant noter que plus le débit de dose diminue plus la mesure est assortie d'une grande incertitude (15\% pour un débit inférieur à $1 \mu \mathrm{Sv} \cdot \mathrm{h}^{-1}$ pour le détecteur utilisé). La contribution à l'ajustement des points très tardifs est donc entachée d'une plus grande incertitude.

En revanche, nos résultats sont complètement similaires en ce qui concerne les patients bénéficiant d'un traitement multiple par rapport aux valeurs de Barrington.

2. À la lumière de ces premières mesures, nous pouvons constater, à la différence des résultats de Barrington, que la distinction entre les patients $\mathrm{P}$ et $\mathrm{M}$ n'est pas opportune. Cependant, il convient une nouvelle fois de remarquer qu'une confirmation par inclusion d'un plus grand nombre de patient ainsi que des mesures tardives supplémentaires sont nécessaires.

3. La méthodologie employée pour cette étude est perfectible puisqu'elle s'appuie sur une modélisation de condition d'exposition bien éloignée de la réalité. Elles ont cependant l'avantage d'être des valeurs hautes; maximisant toujours les temps de contact avec l'entourage. Ainsi, même si la période effective réelle d'un patient donné est supérieure à notre valeur moyenne ; les hypothèses de durée de contact compenseront totalement ou en partie l'erreur sur les durées des périodes de restriction.

4. Malgré la présence d'une fixation extra-cervicale surrénalienne chez une patiente, il n'a pas été constaté de différence de période effective calculée $(13 \mathrm{~h}$ chez cette patiente) par rapport à la valeur moyenne obtenue dans cette étude. Il est dans certains cas difficile de séparer la contribution de l'activité digestive d'une éventuelle fixation métastatique abdominale. Comme nous l'avons déjà mentionné, cette difficulté aurait pu être levée, en partie, par des mesures à plus longue distance du jour d'administration.

5. Les résultats que nous obtenons ne présentent qu'une valeur indicative. Le médecin nucléaire et l'expert en physique médicale peuvent adapter à loisir les durées de restriction de contact en fonction de la situation particulière du patient. Ainsi, la publication 73 de la CIPR (ICRP, 1996) souligne que les 
parents et les proches qui soutiennent et réconfortent les patients peuvent être exposés à des niveaux plus élevés (quelques millisieverts par visite) que ceux mentionnés dans notre travail.

\section{Conclusion}

La disparité (suivant les études suscitées) des recommandations données au patient après son traitement montre qu'il n'est pas aisé d'adopter une méthodologie unique pour déterminer les durées de restriction de contact. Malgré notre méthodologie largement inspirée de celle de Barrington, nous n'obtenons pas des résultats en tout point similaires. L'étude doit être complétée par un plus grand nombre de patients ainsi qu'un ajout de points de mesure plus tardifs. Il n'en reste pas moins qu'en attendant une législation adéquate en France, il appartient au médecin nucléaire et à l'expert en physique médicale de fournir des indications tenant compte ou non de la particularité du mode de vie du patient afin d'exposer au minimum son entourage immédiat.

L'intérêt de notre démarche est qu'elle prend intrinsèquement en compte la quantité de radioélément présente à la sortie du patient au moyen d'une mesure fiable qu'est le débit de dose.

Nous montrons dans cette étude qu'une hospitalisation longue (équivalente à environ une semaine), telle qu'elle est pratiquée dans bon nombre d'institution, est sans doute un peu excessive. Le patient et l'institution d'hospitalisation y gagneraient si une sortie du patient au bout d'un temps plus court (3 jours) était pratiquée. Cette sortie doit être assortie d'une feuille explicative, telle que celle figurant dans le rapport «Radioprotection 97 » de la Commission de la communauté européenne (1999), et détaillée sur les périodes de restriction de contact vis-à-vis de l'entourage du patient. Ce dernier aspect est mis en application en routine dans notre institution et donne entière satisfaction.

Une étude similaire (en conservant l'intérêt de personnalisation des recommandations) est en cours sur les patients traités pour hyperthyroïdie. Cela est d'autant plus important que dans notre institution, entre autres, ces patients sont traités en ambulatoire. Cet aspect ajouté à une période effective associée à cette pathologie nettement plus importante (Gertrud et al., 1996 ; Bockisch et al., 1993), rend obligatoire une information précise au patient.

À ce titre, sur la base de la méthodologie de cette étude, nous avons créé une application qui, en particulier, calcule et donne une information de radioprotection personnalisée le jour de l'injection (et donc de sortie) de l'activité thérapeutique. 


\section{RÉFÉRENCES}

Arrêté (2004) arrêté du 21 janvier 2004 relatif à l'information des personnes exposées aux rayonnements ionisants lors d'un acte de médecine nucléaire.

Barrington S.F., Kettle A.G., O’Doherty M.J., Wells C.P., Somer E.J.R., Coakley A.J. (2002) Radiation dose rates from patients receiving iodine-131 therapy for carcinoma of the thyroid, Eur. J. Nucl. Med. 23, 123-130.

British Institute of Radiology (1999) Working party of the Radiation Protection Committee Patients leaving hospital after administration of radioactive substances, $\mathrm{Br}$. J. Radiol. 72, 121-125.

Bockisch A., Jamitzky T., Derwanz R., Biersack H.J. (1993) Optimized dose planning of radioiodine therapy of benign thyroidal diseases, J. Nucl. Med. 34, 1632-1638.

Commission de la communauté européenne (1999) Radioprotection 97. Radioprotection à la suite d'une thérapie à l'iode 131 (expositions dues aux patients externes ou aux patients hospitalisés sortants). Direction générale Environnement, sécurité nucléaire et protection civile.

Commission of the European Communities (1996) Basic safety standards for the health protection of the general public and the workers against the dangers of ionising radiation. Council directive 96/29 Euratom, Brussels.

Commission of the European Communities (1997) Health protection of individuals against the dangers of ionising radiation in relation to medical exposure. Council directive 97/43, Brussels.

Culver C.M., Dworkin H.J. (1992) Radiation safety considerations for post-iodine-131 thyroid cancer therapy, J. Nucl. Med. 33, 1402-1405.

Décret (2002) décret $n^{\circ}$ 2002-460 du 4 avril 2002 relatif à la protection générale des personnes contre les dangers des rayonnements ionisants.

Décret (2003) décret $n^{\circ}$ 2003-296 du 31 mars 2003 relatif à la protection des travailleurs contre les dangers des rayonnements ionisants.

Gates V.L., Carey J.E., Siegel J.A., Kaminski M.S., Wahl R.L. (1998) Nonmyeloablative iodine-131 anti-b1 radioimmunotherapy as outpatients therapy, J. Nucl. Med. 39, 1230-1236.

Gertrud E.B., Michanek A.M.K., Holmberg E.V.C., Fink M. (1996) Iodine-131 treatment of hyperthyroidism: significance of effective half-life measurements, J. Nucl. Med. 37, 228-232.

Guedj E., de la Forte C., Poinsot L., Bourrely M. (2003) Exposition du public et de l'entourage après traitement d'un patient par $3,7 \mathrm{GBq}$ d $^{131}$ I pour un cancer thyroïdien différencié, Médecine Nucléaire - Imagerie Fonctionnelle et Métabolique 27, 649-654.

ICRP Publication 53 (1988) Radiation dose to patients from radiopharmaceuticals, Ann. ICRP 18, 261.

ICRP Publication 60 (1991) 1990 Recommendations of the International Commission on Radiological Protection, Ann. ICRP 21 (1-3).

ICRP Publication 73 (1996) Radiological protection and safety in medicine, Ann. ICRP 26 (2).

Monsieurs M., Thierens H., Dierckx R.A., Casier K., De Baere E., De Ridder L., De Saedeleer C., De Winter H., Lippens M., Imschoot S., Wulfrank D., Simons M. (1998) Real-life radiation burden to relatives of patients treated with iodine-131: a study in eight centres in flanders (belgium), Eur. J. Nucl. Med. 25, 1368-1376.

Siegel J.A., Marcus C.S., Sparks R.B. (2002a) Calculating the absorbed dose from radioactive patients: The line-source versus point-source model, J. Nucl. Med. 43, 1241-1244.

Siegel J.A., Kroll S., Regan D., Kaminski M.S., Wahl R.L. (2002b) A practical methodology for patient release after tositumomab and 131I-tositumomab therapy, J. Nucl. Med. 43, 354-363. 